\title{
Directional Self-Learning of Genetic Algorithm
}

\author{
Lin Cong \\ Institute of Intelligent Information Processing, \\ Xidian University \\ Xi'an, 710071 China \\ conglin01@yahoo.com.cn \\ Licheng Jiao \\ Institute of Intelligent Information Processing, Xidian \\ University \\ Xi'an, 710071 China \\ Ichjiao@mail.xidian.edu.cn
}

\begin{abstract}
In order to overcome the low convergence speed and prematurity of classical genetic algorithm, an improved method named directional self-learning of genetic algorithm (DSLGA) is proposed in this paper. Through the self-learning operator directional information was introduced in local search process. The search direction was guided by the false derivative of the function fitness. Using the four operators among the individuals, the best solution was updated continuously. In experiments, DSLGA was tested on 4 unconstrained benchmark problems, and the results were compared with the algorithms presented recently. It showed that DSLGA performs much better than the other algorithms both in the quality of the solutions and in the computational complexity.
\end{abstract}

\section{Categories and Subject Descriptors:}

G.1.6 [numerical analysis]: Optimization. Global optimization, Unconstrained optimization.

\section{General Terms:}

Algorithms, Measurement, Performance Design

Keywords: genetic algorithm directional self-learning numerical optimization evolutionary computation.

\section{INTRODUCTION}

GAs are adaptive heuristic search algorithm premised on the evolutionary ideas of natural selection and genetic. The major drawback of GAs is that although they may be efficient in locating the basins of the optima, they are often unable to explore these basins effectively and quickly in order to find the exact global optimization with a high degree of accuracy and within a small number of generations. To cope with this inefficiency, several hybrid genetic schemes have been suggested, such as microgenetic algorithm(MGA) [1], orthogonal genetic algorithm(OGA/Q) [2], multiagent genetic algorithm(MAGA) [3], fast evolutionary programming(FEP) [4], and so on. These algorithms proved to be effective and boosted the development of genetic algorithms.

In recent years, researchers have realized that the combination of GAs with local search techniques can improve the quality of the

Copyright is held by the author/owner(s).

GECCO'05, June 25-29, 2005, Washington, DC, USA.

ACM 1-59593-010-8/05/0006.

\author{
Yuheng Sha \\ Institute of Intelligent Information Processing, \\ Xidian University \\ Xi'an, 710071 China \\ shayuheng@163.com \\ Fang Liu \\ School of Computer Science and Technology, \\ Xidian University \\ Xi'an, 710071 China \\ f63liu@163.com
}

solving problems on numerical optimizations. There are many methods on local search techniques. Reference [1] introduced an application of microgenetic algorithm and obtained better performance. Enlightened by them, this paper integrates microgenetic algorithm with GAs to form a new algorithm, directional self-learning of genetic algorithm (DSLGA), for solving the global numerical optimization problems.

\section{THE CONSTRUCTION OF THE DIRECT-IONAL FALSE DERIVATIVE}

In GAs, the crossover strategy will make the candidate solutions in the random position, the search speed decreased accordingly. The directional false derivative is presented which uses the linear function to approximate the search direction. The global numerical optimization can be formulated as the following objective function

$$
\min f(x), x=\left(x_{1}, x_{2}, \cdots, x_{n}\right) \in S
$$

where $S \subseteq R^{n}$ defines the search space which is an ndimensional space bounded by the parametric constraints $\underline{x}_{i} \leq x_{i} \leq \bar{x}_{i}, i=1,2 \cdots ; n$. Thus, $S=[\underline{x}, \bar{x}]$, where $\underline{x}=\left(\underline{x}_{1}, \underline{x}_{2}, \cdots, \underline{x}_{n}\right)$ and $\bar{x}=\left(\bar{x}_{1}, \bar{x}_{2}, \cdots, \bar{x}_{n}\right)$. Under the condition of without prior knowledge, we can use the fitness values to estimate the search direction.

If the search direction can be approximated using the linear function, the normal search direction can be showed that

$$
d=\left(f i t\left(x_{i}\right)-f i t\left(\min _{i}\right)\right) / f i t\left(x_{i}\right)
$$

where $f i t\left(\min _{i}\right)$ and $f i t\left(x_{i}\right)$ are the minimum fitness and the individual fitness respectively.

\section{THE DIRECTIONAL SELF-LEARNING OPERATOR}

Hill-climbing operators in GAs are employed in [5], [6] for solving the continuous variable problems. Self-learning operator realizes its local search in the micro population genetic algorithm. In the self-learning operator, first of all, the self-learning population, $L$, is generated. The size of the $L$ is $L_{\text {size }}$, and all the individuals, $L_{i}=\left(L_{i, 1}, L_{i, 2}, \cdots, L_{i, n}\right), i=1,2, \cdots, L_{\text {size }}$ where $n$ is the dimension of the function, are generated according to

$$
L_{i}= \begin{cases}x_{i} & i=1 \\ \text { New }_{i} & \text { others }\end{cases}
$$


where $N_{e w}=\left(e_{i, 1}, e_{i, 2}, \cdots, e_{i, n}\right)$ is determine by the equation as follows.

$$
e_{i, k}=x_{i, k} \cdot U(1-\text { sradius }, 1+\text { sradius })
$$

where sradius $\in[0,1]$ represents the search radius.

Let $\operatorname{Max}_{i}=\left(m_{1}, m_{2}, \cdots, m_{n}\right)$ is the maximum fitness individual among $L$, namely, $\forall a \in L \quad f i t(a) \leq f i t\left(\operatorname{Max}_{i}\right)$. If $L_{i}$ satisfies (5), it is a winner; otherwise it is a loser.

$$
\operatorname{fit}\left(L_{i}\right)>\operatorname{fit}\left(\operatorname{Max}_{i}\right)
$$

If $L_{i}$ is a winner, it can still live in the population. If $L_{i}$ is a loser, it must die, and it will be occupied by $\operatorname{Max}_{i}$. $\operatorname{Max}_{i}$ has two strategies to occupy the $L_{i}$, and it select them with probability $p_{0}$. If $U(0,1)<p_{0}$, occupying strategy 1 is selected; otherwise occupying strategy 2 is selected. In the two occupying strategies, $\operatorname{Max}_{i}$ first generates a new individual, $\operatorname{New}_{i}^{1}=\left(e_{i, 1}^{1}, e_{i, 2}^{1}, \cdots, e_{i, n}^{1}\right)$, and then $N e w_{i}^{1}$ is used to occupy the $L_{i}$.

In occupying strategy 1 , using the linear approximate direction $d$ can direct to find better solutions and shorten the search time to improve the convergence speed. New $w_{i}^{1}$ is determined by,

$$
e_{i, k}^{1}=m_{i, k}+d^{*}\left(m_{i, k}-L_{i, k}\right) \quad \mathrm{k}=1,2, \cdots, \mathrm{n}
$$

In occupying strategy $2, N e w_{i}^{1}$ is first mapped on $[0,1]$ according to the equation (7).

$$
m_{i}^{\prime}=\left(m_{i}-\underline{x}\right) /(\bar{x}-\underline{x}) \quad i=1,2, \cdots, n
$$

Then $\operatorname{New}_{i}^{\prime}=\left(\dot{e}_{i, 1}^{\prime}, e_{i, 2}^{\prime}, \cdots, e_{i, n}^{\prime}\right)$ is determined by

$$
\text { New } i_{i}^{\prime}=\left(m_{1}^{\prime}, m_{2}^{\prime}, \cdots, m_{i_{1}-1}^{\prime}, m_{i_{2}}^{\prime}, m_{i_{2}-1}^{\prime}, \cdots, m_{i_{1}+1}^{\prime}, m_{i_{1}}^{\prime}, m_{i_{2}+1}^{\prime}, m_{i_{2}+2}^{\prime}, \cdots, m_{n}^{\prime}\right)
$$

where $1<i_{1}<n, 1<i_{2}<n, i_{1}<i_{2}$. Finally, New is obtained by mapping $N e w_{i}^{1}$ back to $[\underline{x}, \bar{x}]$ according to

$$
e_{i, k}^{1}=\underline{x}+e_{i, k}^{\prime} \cdot(\bar{x}-\underline{x}) \quad k=1,2, \cdots, n
$$

Then the new mutation individual, $\operatorname{New}_{i}^{2}=\left(e_{i, 1}^{2}, e_{i, 2}^{2}, \cdots, e_{i, n}^{2}\right)$, is obtained according to the follows.

$$
e_{i, k}^{2}=e_{i, k}^{1}+G(0,1 / t)
$$

where $G(0,1 / t)$ is a Gaussian random number generator, and $t$ is the evolution generation. Then, $x_{i}$ is replaced by $\mathrm{New}_{i}^{2}$.

\section{EXPERIMENTAL STUDIES ON GLOBAL NUMERICAL OPTIMIZATION}

Here some benchmark functions are adopted to test the performance of DSLGA.

$F_{1}$-Minimize $f(\boldsymbol{x})=\sum_{=1}^{n-1}\left[100\left(x_{i+1}-x_{i}^{2}\right)^{2}+\left(x_{i}-1\right)^{2}\right], \mathrm{S}=[-30,30]^{\mathrm{n}}, \mathrm{x}^{*}=(1,1, \cdots, 1), \mathrm{f}\left(\mathrm{x}^{*}\right)=0$.

$F_{2}$-Minimize $f(\boldsymbol{x})=\sum_{i=1}^{n}\left(-x_{i} \sin \left(\sqrt{\left|x_{i}\right|}\right)\right) \quad, \quad \mathrm{S}=[-500,500]^{\mathrm{n}}$,

$\boldsymbol{x}^{*}=(420.9687,420.9687, \ldots, 420.9687), f\left(\boldsymbol{x}^{*}\right)=-12569.5$.

$F_{3}$-Minimize $f(x)=\frac{\pi}{n}\left\{10 \sin ^{2}\left(\pi y_{1}\right)+\sum_{i=1}^{n-1}\left(y_{i}-1\right)^{2}\left[1+10 \sin ^{2}\left(\pi y_{i+1}\right)\right]+\left(y_{n}-1\right)^{2}\right\}$

$+\sum_{i=1}^{n} u\left(x_{i}, 10,100,4\right), \mathrm{S}=[-50,50]^{\mathrm{n}}, \mathrm{x}^{*}=(1,1, \cdots, 1), \mathrm{f}\left(\mathrm{x}^{*}\right)=0$. where

$u\left(x_{i}, a, k, m\right)=\left\{\begin{array}{ll}k\left(x_{i}-a\right)^{m} & x_{i}>a \\ 0 & -a \leq x_{i} \leq a \\ k\left(-x_{i}-a\right)^{m} & x_{i}<-a\end{array}, \quad y_{i}=1+\frac{1}{4}\left(x_{i}+1\right)\right.$.

$F_{4}$-Minimize $f(x)=\frac{1}{10}\left\{\sin ^{2}\left(3 \pi x_{1}\right)+\sum_{i=1}^{n-1}\left(x_{i}-1\right)^{2}\left[1+\sin ^{2}\left(3 \pi x_{i+1}\right)\right]\right.$ $\left.\left.+\left(x_{n}-1\right)^{2}\left[1+\sin ^{2}\left(2 \pi x_{n}\right)\right]\right\}+\sum_{i=1}^{n} u\left(x_{i}, 5,100,4\right), \mathrm{S}-50,50\right]^{\mathrm{n}}, \mathrm{x}^{*}=(1,1, \cdots, 1)$, $f\left(\boldsymbol{x}^{*}\right)=0$.

The experiment studies the performance of DSLGA on functions with 30 dimensions. The termination criterion of DSLGA is one of the objectives, $\left|f_{\text {best }}-f_{\min }\right|<\varepsilon$, is achieved, where $f_{\text {best }}$ and $f_{\text {min }}$ represent the best solution found until the current generation and the global optimum, respectively. To consistent, $\varepsilon=10^{-3}$ and the maximum generation being 2000 are used for all functions. In the following experiments, the parameter settings are: $\mathrm{N}=100, p_{\mathrm{c}}=0.9$, $p_{\mathrm{m}}=0.1, s L_{\text {size }}=10, p_{0}=0.2$, sradius $=0.2$, sgen $=10$.

Table 1. The comparisons between FEP and DSLGA

\begin{tabular}{|c|c|c|c|c|c|}
\hline \multirow{2}{*}{$\boldsymbol{f}$} & \multirow{2}{*}{$\boldsymbol{f}_{\min }$} & \multicolumn{2}{|c|}{$\begin{array}{c}\text { Mean value } \\
\text { (standard deviation) }\end{array}$} & \multicolumn{2}{c|}{ Mean function value } \\
\cline { 2 - 6 } & $\mathbf{F E P}$ & DSLGA & FEP & DSLGA \\
\hline$F_{1}$ & 0 & $\begin{array}{c}5.06 \\
5.87\end{array}$ & $\begin{array}{c}5.395 \times 10^{-4} \\
\left(3.187 \times 10^{-4}\right)\end{array}$ & 2000000 & 111635 \\
\hline$F_{2}$ & -12569.5 & $\begin{array}{c}-12554.5 \\
(52.6)\end{array}$ & $\begin{array}{c}-12569.46 \\
\left(8.784 \times 10^{-3}\right)\end{array}$ & 900000 & 124481 \\
\hline$F_{3}$ & 0 & $\begin{array}{c}9.2 \times 10^{-6} \\
\left(3.6 \times 10^{-6}\right)\end{array}$ & $\begin{array}{c}7.825 \times 10^{-7} \\
\left(2.505 \times 10^{-7}\right)\end{array}$ & 150000 & 38402 \\
\hline$F_{4}$ & 0 & $\begin{array}{c}1.6 \times 10^{-4} \\
\left(7.3 \times 10^{-5}\right)\end{array}$ & $\begin{array}{c}7.820 \times 10^{-5} \\
\left(2.515 \times 10^{-5}\right)\end{array}$ & 150000 & 12388 \\
\hline
\end{tabular}

\section{CONCLUSION}

An improved genetic algorithm based on directional self-learning operator was proposed in this paper. It can obtain the optimization by competition, cooperation and self-learning operators. In experiments, benchmark problems are used to test the performance of DSLGA and the results compared with FEP. The comparison results show that the method presented in this paper has not only global but also local search abilities and can avoid prematurity while maintain the diversity of the population.

\section{REFERENCES}

[1] Kazarlis S A, Papadakis S E, Theocharis J B, Petridis V. Microgenetic algorithms as generalized hill-climbing operators for GA optimization. IEEE Trans. Evolutionary Computation.2001,5(3):204-217.

[2] Leung Y W, Wang Y. An orthogonal genetic algorithm with quantization for global numerical optimization. IEEE Trans. Evolutionary Computation. 2001, 5(1): 41-53.

[3] Zhong Weicai, Liu Jing, Xue Mingzhi, Jiao Licheng. $A$ multiagent genetic algorithm for global numerical optimization. IEEE Trans. System, Man, and CyberneticsPart B. 2004, 34(2): 1128-1141.

[4] Xin Yao,Yong Liu, Guangming Lin. Evolutionary programming made faster. IEEE Trans. Evolutionary Computation. 1999, 3(2):82-102.

[5] S. A. Kazarlis, A. G. Bakitzis, V. Petridis, A genetic algorithm solution to the unit commitment problem. IEEE Trans. Power Syst. 1996, 11(1) 83-92.

[6] V. Petridis, S. Kazarlis, A. Bakirtzis, Varying fitness functions in genetic algorithm constrained optimization: The cutting stock and unit commitment problems. IEEE Trans. Syst, Man, Cybern. B, vol.28,pp.629-640,Oct.1998. 